\title{
An output spooling system for continuous data-logging paradigms
}

\author{
HOWARD L. KAPLAN \\ Clinical Research Unit, Addiction Research Foundation of Ontario \\ 33 Russell Street, Toronto, Ontario, Canada MSS 2S1
}

\begin{abstract}
Spooling is the process of temporarily routing data to high-speed disk storage, en route between programs and peripherals of different speeds. In the real-time laboratory, such mismatches may occur between input data rates from sensors, processing programs, and archival storage on magnetic tape. To handle these situations, a continuous real-time data spooling system is now under development in our laboratory. Running under a multiprogramming executive, a spooling dispatcher schedules asynchronous device drivers and data manipulation programs in an attempt to maximize throughput without any loss of data.
\end{abstract}

The Human Responses Laboratory of the Addiction Research Foundation of Ontario is designed to be shared among experimenters of diverse interests. As we reviewed the protocols for some of the experiments planned for the laboratory's first year of operation, several experiments appeared to have a common need to digitize, process, and record analog input data at a fairly high rate, and for durations that would exceed the capacity of our disk storage. Many of these can record their data on magnetic tape in real or near-real time, providing a mechanism is available to cope with speed variability in the input data rate, the processing programs, and the tape writing rate. A general-purpose spooling system is being built to cope with these demands.

\section{WHAT IS SPOOLING?}

The word "spool," in the sense in which it is used in computing, is often treated as an acronym. I had always considered it to be a rather obvious metaphor: A linear stream of data is wound around a circular device, such as a disk pack or tape reel, and later unwound. However, in the Encyclopedia of Computer Science (Taylor, 1976), "SPOOL" refers to "Simultaneous Peripheral Operation, On-Line." This use seems to have originated among IBM users, because the iden. tical definition appears in a computer glossary published by SHARE. For rather obvious commercial reasons, the Varian computer systems use a different explanation of the acronym, "Simultaneous Peripheral Output OverLap."

There are definite advantages to using "SPOOL" as an acronym instead of a metaphor. In the oldest sense of the word, a core upon which thread or wire is wound, a spool is a perfect example of a device which, although physically round, operates logically like a stack, a last-in/first-out device. In contrast, computer spooling systems must preserve the first-in/ first-out order of the data being moved to peripheral devices, at least within individual tasks, though recent high-priority output may occur before older low-priority output from another task.

Spooling systems can generally be divided into two classes, batch and continuous. Batch spooling is commonly used on medium- and large-scale computer systems. Data is not transferred directly between applications programs and relatively slow peripherals such as card readers and line printers; instead, the entire input deck or output listing is written to relatively fast disk or drum storage, which can communicate with the applications program at a much higher data transfer rate. Large applications programs need not occupy memory while their slow I/O operations occur; instead, they can be loaded only when the input data is ready and exit as soon as processing is complete, leaving only a much smaller spooling program resident to perform the data transfers to printers or punchedcard devices.

In the real-time laboratory, batch spooling can also be a useful technique. One obvious application is to allow listing of the printed output from program compilation, text editing, and similar applications to occupy one hard-copy terminal while another $\mathrm{I} / \mathrm{O}$ device controls a real-time experiment. Even if the computer cannot support enough memory for simultaneous compilation and control, it can often support the much smaller memory requirement (including a smaller executive control program) for simultaneous real-time control and batch printing of disk files. More important for real-time work, batch spooling can be used to log data from fast sensors directly to disk, only later reading it back into memory for transfer to its ultimate destination, which may be an analysis program or slower (but cheaper and larger) tape storage. Whenever the disk capacity and the experimental data rates allow such a solution, it is the simplest form of spooling to implement. 


\section{REAL-TIME SPOOLING}

In contrast to these relatively simple situations, experiments may arise where there is not enough disk capacity to record all of the data that will arrive in one continuous stream, but where there is not enough main memory to buffer all of the speed variations among input sensors, data manipulation programs, and output tape storage. For example, we have what sounds like a large amount of disk capacity to devote to receiving digitized analog data, 2.5 million words. If a 16-channel EEG records at $1,000 \mathrm{samples} / \mathrm{sec} / \mathrm{channel}$, the disk is filled in less than $3 \mathrm{~min}$. If we want to digitize a single speech channel at 40,000 samples/sec, the disk is filled in $1 \mathrm{~min}$. Other biologic signals, such as EMG or EKG recordings, may require similar high data input rates. For these signals, a spooling system for records of several minutes' duration must be a continous spooling system, one which can begin the process of moving some data to its final destination, tape or an analysis program, while more data is still being collected.

Why is a spooling system necessary at all? If the experimental protocol has high burst rates of data separated by periods of no data, and if the tape can - cope with the average rate but not the burst rate, then spooling can bridge this discrepancy. For example, our tape drive can record data at about 25,000 words/ sec. If speech is digitized at 40,000 samples/sec, the tape cannot store it in real time. If data is diverted to disk as it is collected, alternating 1-min speech periods with 1-min silent ones, and if data is moved to tape as fast as the tape is ready for it, then we can spread each minute's data collection over 2 min of recording.

Another reason for using continuous spooling is the unreliability of tape drives. Based on experience with an off-site analog-to-digital (A/D) conversion facility, there are often instances when the tape drive must rewrite the same tape record several times to overcome faults in the tape. Even with reliable hardware, it may sometimes be necessary to insert labels, endof-file marks, or other nondata items into the output data stream. These interruptions may have only a slight effect on the mean rate at which data can be moved to tape, but they may greatly lower the maximum safe rate of data collection unless the data can be moved to temporary disk storage en route to tape. In addition, there may be software components which lead to variability in the rate at which the tape needs to collect data. For example, in a speechprocessing system, there may be a program to remove the silent intervals from the speech record, replacing them with a special marker to indicate their duration. This postprocessing may lower the mean rate of data transfer to tape to a value below the tape's maximum capacity, but there is again the problem of burst rates. There may even be a problem of burst rate capacity at the input sensor stage. For instance, a timer that records each interevent interval as one data word requires higher throughput at times of high activity than at times of little activity.

These problems are not peculiar to high data rate protocols. They may also arise in a low-throughput system with a floppy disk and a cassette recorder, interfaced to a microprocessor with limited memory. The critical condition suggesting a need for spooling is a variability in the input or output worst-case data transfer rate that is large compared to the capacity to save data in main memory.

Though we have done little formal analysis of the effectiveness of spooling, some limitations are clear. Most obviously, the data backlog cannot exceed the disk capacity. To assess throughput, the data pipeline is divided into two parts: those tasks that must be performed before the data is spooled to disk and those performed after it is returned from disk. The throughput of the postdisk stages cannot exceed the difference between the disk throughput rate and the mean data rate. In other words, if the disk capacity is 80,000 words/sec and if data is being put onto disk at 50,000 words/sec, then the disk cannot supply data to subsequent tasks faster than 30,000 words/sec as long as input data is still arriving. When the disk is twice as fast as the input data rate, it will not be the limiting factor in the system throughput.

\section{A FAMILIAR ANALOG OF CONTINUOUS SPOOLING}

The simplest form of a continuous spooling system is the interrupt-driven circular Teletype output buffer. This kind of system uses main memory in the same way that a true spooling system can use disk storage, and displays many of the principles used in data spooling systems for real-time applications. There are three software components, a data buffer, a type-or-save program, and an interrupt handler. The buffer is simply a contiguous block of memory, such as 32 words, where each word is accessed individually. Two pointers are used to point to the words, a storage pointer associated with the type-or-save program and a retrieval pointer associated with the interrupt handler. Each pointer accesses successive buffer words. When the pointer passes the end of the buffer, it is reset to the beginning of the buffer; it is in this sense that the buffer is termed "circular." Figure 1 shows such a buffered output system in the process of printing a brief message.

When the program is initialized, both pointers access the beginning of the buffer, and all words are set to the "empty" state, containing zero. Whenever the applications program needs to type a character, it passes that character to the type-or-save routine. If the printer is not busy typing another character, then 


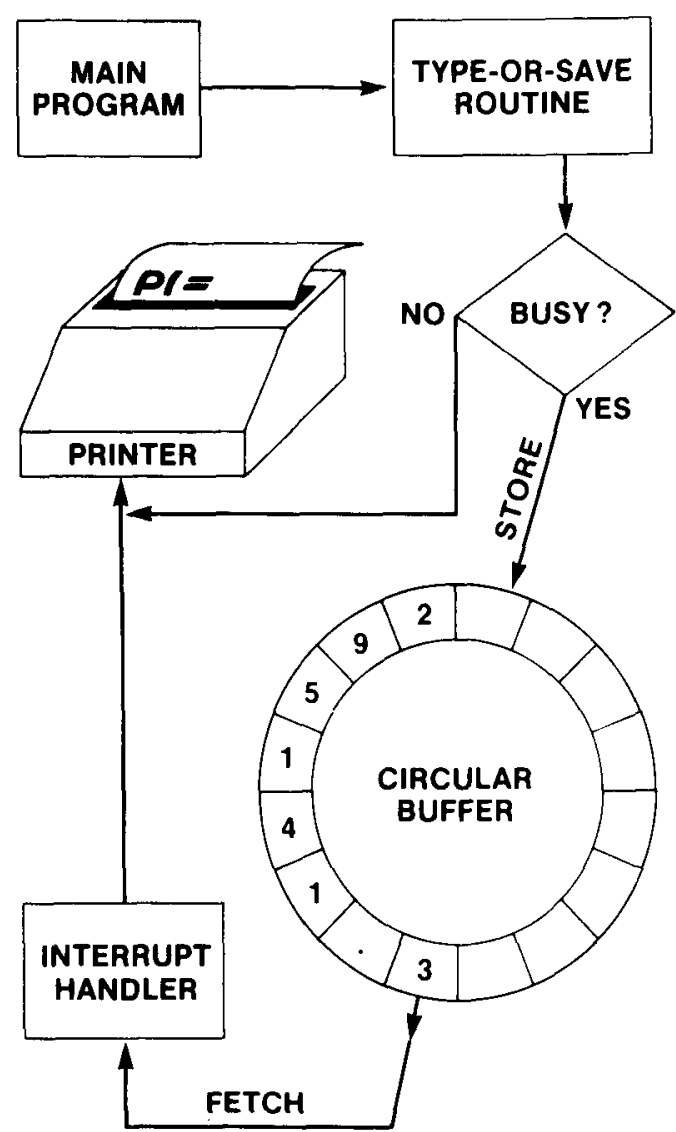

Figure 1. An interrupt-driven Teletype driver with a circular buffer. Using the store pointer, the type-or-save routine fills the buffer with characters whenever the printer is busy. Using the fetch pointer, the interrupt handler removes characters from the buffer until it is empty, then resets the printer busy flag.

the character is sent to the printer immediately and a flag is set to "busy." If the printer is busy when a character is passed to the type-or-save routine, the character is saved where the storage pointer is pointing, and the pointer is incremented (circularly). However, if that storage location is already occupied, the subroutine simply loops until that storage location is free.

The interrupt handler responds to the signals the printer interface sends as it completes the typing of each character. When such an interrupt is issued, the handler inspects the buffer location accessed by the retrieval pointer. If the location is empty, the printer flag is simply set to "not busy." If the location is not empty, the character in that location is sent to the printer, the location is emptied, and the pointer is incremented (circularly). If the type-or-save routine is looping until the buffer location is empty, it can store its character as soon as the interrupt handler returns control to the interrupted type-or-save routine.

A continuous spooling system is analogous to a circular character-buffering system. Instead of single characters being saved, entire data records are saved, and the circular buffer medium is not main memory, but disk. However, the basic principle, that of consecutive locations (disk sectors) being reused as temporary data-holding areas, remains the same.

\section{SOME DEFINITIONS}

A block is a fixed number of consecutive data points. The data points travel through the spooling system together and are treated as a unit. The blocks can be numbered consecutively from 1 . If block size is 1,000 , then the first 1,000 points to be collected will also be the first block to be written to tape, followed by the second 1,000 points. In theory, a continuous spooling system can pass an infinite number of blocks of data; in practice, the finite size of the tape reels (and finite future age of the universe) limits it.

A buffer is a set of contiguous memory locations used to hold a single block of data while it is in the computer. The number of such buffers is rather small, perhaps 5 to 15 , depending on the memory available, the block length, the disk speed, and other system tuning considerations. While a given block of data stays in memory, it occupies the same buffer; if it is written to disk and then read back into memory, it may be read into a different buffer.

The spooling dispatcher is the program that assigns blocks to buffers, requests the initiation of I/O transfers, determines which disk tracks are to be used to hold data blocks, and otherwise controls the operations of the spooling system. In a nondedicated system, the spooling dispatcher is an applications program, different from the operating system dispatcher that resolves priority conflicts, schedules interrupt handlers, and performs similar tasks. The spooling dispatcher makes frequent demands upon the operating system dispatcher.

A processing program is an auxiliary task, running asynchronously from the spooling dispatcher, that operates on the blocks of data in their buffers. Such a program may move data between buffers and peripheral hardware or may simply process the data in memory, rearranging it within a block or printing warning or summary messages about it. It is useful to consider the tasks of moving data to and from disk as being separate processing programs, even though they may invoke a common system disk handler. The spooling system always includes processing programs to move data into memory from the $A / D$ converter, to disk, from disk, and to tape, and to postprocess the data buffer before it is written to tape. This postprocessing program might delete uninteresting parts of the record, remove artifacts, rearrange the order of data points to separate different input channels, or collect summary statistics to be printed in real time. Other spooling systems might include programs to preload the buffer with stimulus material to be output 
before the responses are input or to summarize the data instead of sending it on to tape.

A role represents the current state of any block of data. A role is associated with each processing program, and roles are also associated with waiting for processing programs to complete, with being stored on disk, and with being stored safely on tape.

\section{THE SPOOLING PIPELINE}

Figure 2 depicts the 12 roles a block may occupy in our spooling system as it progresses through the spooling pipeline. Five of the roles indicate blocks being processed by other programs, and the rest indicate conditions of waiting to occupy subsequent roles. The following list explains the roles in more detail: (1) The block will be the next data input block. A previously inactive buffer has been assigned to this block, and it will occupy Role 2 as soon as the current block has been filled with data. (2) The block is currently receiving data from the $A / D$ converter. When

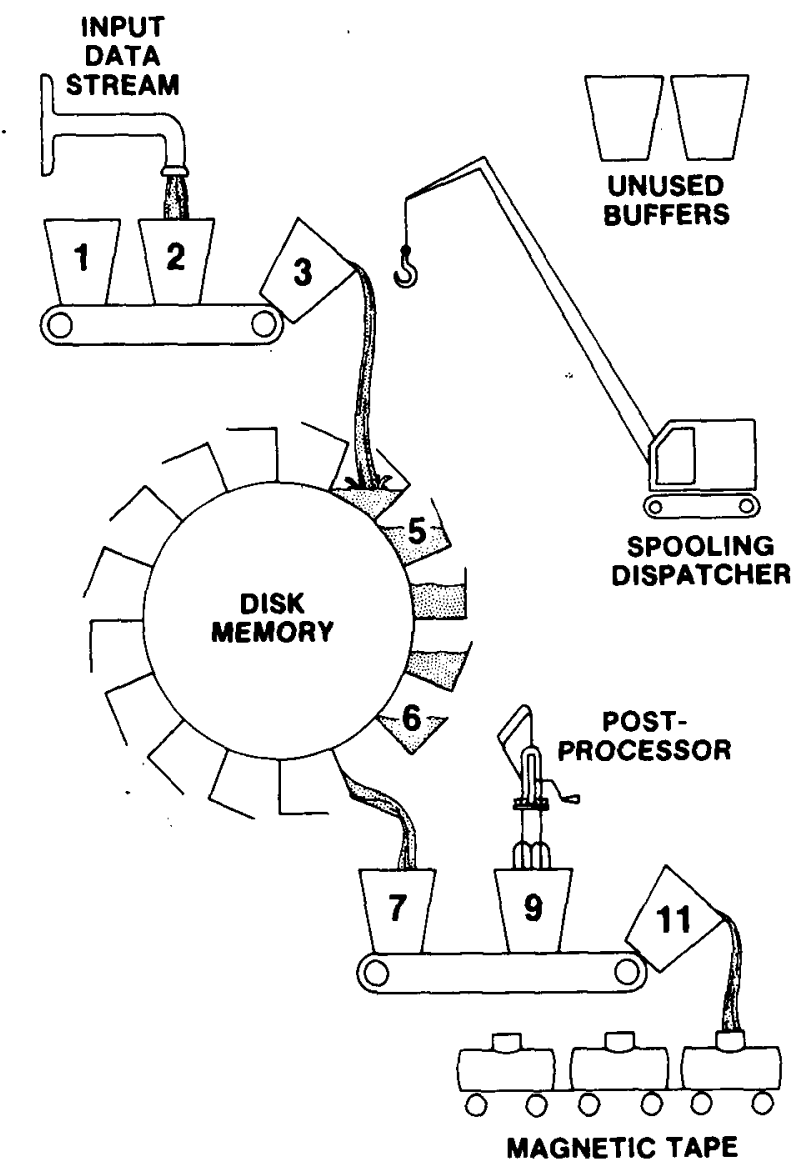

Figure 2. Data enters the spooling pipeline from the $A / D$ converter and is stored in a buffer. If the postprocessor is not busy, the spooling dispatcher lets it process the data block in the same buffer in which it was collected. If the postprocessor is busy, the spooling dispatcher diverts the data to disk. Role numbers are shown for the significant data blocks. the transfer is complete, it will occupy Role 8 if there is no backlog of blocks waiting to be processed by the inspection program, and Role 3 otherwise. (3) The block is being written to disk. If Role 9 becomes vacant while the transfer is occurring and if there is no backlog, then this block and buffer will be moved immediately to Role 9, and the copy on disk will be ignored. (4) The block has been written safely to disk, so that the buffer may be reused if necessary. However, if Role 9 becomes vacant and needs this block while the buffer still con. tains it, the copy on disk will be ignored. (5) The only record of this block is on disk, its buffer has been released for another use, and this is the last block to have been written to disk (but not the only block on disk). (6) The only record of this block is on disk, and this is the first (and perhaps only) record queued to leave disk when the inspection program is ready for it. (7) This block has been reassigned a buffer and is in the process of awaiting transfer from disk to memory. (8) This block is in memory, having just arrived from disk or from Role 2 and is waiting for the postprocessing program to use it. (9) This block is being used by the postprocessing program. (10) The postprocessor has finished with this block, and the block is waiting for the tape output program to accept it. (11) The tape output program is writing this block to tape. (12) The block is stored safely on tape, and its buffer has been released for another block.

\section{THE PROGRAMMING ENVIRONMENT}

The spooling dispatcher is written for the specific requirements of our hardware and software environment. The computer is a Varian V76, under the VORTEX II operating system. This is a multiprogramming, multitasking system, in which explicit priorities are assigned to both applications programs and device drivers. Program priorities are numbered from 0 to 31 , with 31 being the highest. At any moment, the system dispatcher has activated the highest priority program that requires the use of the CPU. That is, any task that is activated is not suspended for a time delay, a hardware interrupt, or resumption by another program (generally a device driver). While the default for applications programs is to wait for $\mathrm{I} / \mathrm{O}$ to complete before resuming, a program can also initiate $\mathrm{I} / \mathrm{O}$ and then continue processing, even initiating another I/O operation to proceed concurrently with the first one. Multiple I/O requests queued to the same peripheral are handled in the order of their calling programs' priorities and in order of request within equal priorities.

High-speed peripheral devices, such as the disk, tape, and $A / D$ converter, transfer data on a cycle-stealing basis through devices known as Buffer Interlace Controllers (BICs). Once a device driver initiates a data transfer through a BIC, it can suspend CPU operations until a transfer-complete interrupt is issued, 
freeing the CPU for other tasks. However, the subsequent data transfer cannot begin until the device driver sends the address of the next data block to the BIC. As supplied by Varian, the device driver programs handle only one block of data at a time and must pass through several layers of system overhead to learn the addresses of the next data buffer, even if the data transfer request is already queued. In order to collect data continuously prior to spooling it, the $A / D$ driver was modified so that each call supplies the addresses of both the current and the subsequent data buffer. The revised driver, which is the highest priority task in the system, gains control of the processor and inhibits all interrupts $1 \mathrm{msec}$ before the expected buffer completion signal will be issued. Without going through the overhead of the interrupt handler, it simply loops to await that completion signal, sends the address of the next buffer in the time interval between two data points, turns the interrupts back on, and exits. Subsequently, the calling program issues an explicit request authorizing the collection of the data block already in progress and passing the address of the buffer to be used next. With this device driver, continuous analog data is collected.

Since the most critical time window in the system is that for resuming analog sampling at the end of each block, the A/D driver has Priority 31 . The next highest priority, 29, is assigned to the disk driver, so that no other program except the $A / D$ driver can inhibit it from initiating the next queued request when the previous one is complete. The spooling dispatcher normally runs at Priority 28 , but occasionally raises its own priority to 29 , so that its requests to write buffers to disk will be queued ahead of its own, lower priority requests to read blocks back from disk. Other device drivers (such as magnetic tape and Teletype), the CPU-bound postprocessing program, and other applications programs (editors, etc.) all run at priorities below 28 .

\section{FILLING THE SPOOLING PIPELINE}

Data collection starts when the spooling dispatcher assigns two buffers to Roles 1 and 2 , one for active data collection and the other waiting to be the next input buffer, and starts the A/D driver program. It then enters its general suspension routine. Whenever any processing program completes its work, it resumes the activity of the spooling dispatcher. Starting at the end of the list of roles, the dispatcher checks each role in turn, testing whether the role is occupied and whether the block can be moved to the next role, generally leaving its current role vacant. For those roles associated with a processing program, the block advances a role only if the program has completed. For other roles, the block advances if the next role in line becomes vacant, with two important exceptions. If the post- processor is able to accept a block from Role 3 or 4 , that block being the next sequential block to be inspected, then the buffer is immediately passed to Role 9, bypassing the need to write the block to disk or ignoring the disk copy if the transfer to disk has already begun. As part of the same strategy, a block in Role 4 gives up its buffer only if the buffer is needed for Role 1 or if there is already a significant backlog on disk, in order to eliminate the need to transfer the block back to memory from disk.

The one important bottleneck in the system is at Role 3 , blocks waiting to be moved to disk. Because of rotational delays, track access delays, and occasional competition from other programs, the disk cannot always collect data at a constant rate. Therefore, it may be necessary to hold data blocks in memory briefly until the disk can accommodate them. It may often occur that a subsequent block is collected from the A/D program before all previous blocks are safely on disk; in such a case, there may be several blocks in main memory, all queued to move to disk. The number of buffers available limits the maximum allowable backlog at this stage. To maximize the number of buffers so available, we limit the number of buffers allocated to the postdisk stages of the system. First, a buffer is allocated for Role 7 only when there are at least two free buffers in the pool, so reading a block back from disk will never take the only buffer potentially available to collect $A / D$ data. In addition, a buffer is not allocated for Role 7 unless Role 8 is already vacant. That is, we do not read a block back from disk until the previous block is being handled by the postprocessing program. At the cost of a potential loss of throughput to tape, this preserves all but three blocks for the predisk stages of the system, while still allowing concurrent postprocessing and tape writing. These decisions about buffer allocation are appropriate to a system in which buffers are a limiting resource; with more main memory available, and total throughput a problem, buffers might be allocated to roles differently.

Whenever the spooling dispatcher moves a block into a role that requires initiation of a separate processing program, the operating system will attempt to resume that processing program. Because of the priority structure, only the $A / D$ and disk drivers will actually begin executing instead of the spooling dispatcher, and they will return control within 1 or $2 \mathrm{msec}$. In particular, the inspection program, which may consume a relatively large amount of CPU time will not actively execute unless the spooling dispatcher is suspended. After completing the role scan, Role 1 , the spooling dispatcher cannot simply suspend until the first processing program signals completion and resumes it. Instead, the spooling dispatcher must briefly turn off the interrupt system while it tests to see if either of the higher priority tasks, the $A / D$ and disk 
drivers, has completed during the scan. If one has, then the role scan is resumed at the top to process that completion; if not, the spooling dispatcher can safely suspend, to be resumed by the first processing task to complete.

From the standpoint of the tasks that are upstream of the disk, we have an output spooling system, one which is collecting data as fast as it can be generated. However, the view from downstream is the opposite. The postprocessor and tape drive see the spooling system as an input spooler. Unlike a batch input spooler, which insures that card images pass to an applications program at a reasonably fast rate, this input spooler insures that data blocks arrive at a reasonably slow rate, no faster than they can be processed.

\section{WHY MULTIPROGRAMMING?}

The spooling system described here is implemented in an explicitly multiprogramming environment. As multiprogramming systems are often expensive, have high overhead in CPU and memory requirements, and impose restrictions on application programs, we can reasonably ask what is gained by using such a system. In particular, could the whole spooling system be implemented more easily with stand-alone dedicated software, without separate device drivers, priorities, and queued $\mathrm{I} / \mathrm{O}$ requests? If this were strictly a datalogging system, with no postprocessing program, then a dedicated system with no operating system supervision might work just as well. The device driver programs, those that initiate and terminate data transfers, are relatively short programs, just a few milliseconds on modern systems. These could easily be incorporated into the code of a role-scanning loop, rather than some of them being deferred to run at lower priority outside the loop. The direct memory access hardware, however, is still required. Except for the problem of restarting analog sampling when each block is complete (a task that could be handled with a more intelligent BIC, that is, in hardware), even the interrupt structure is unnecessary, as the peripheral devices could simply be polled in a continuous loop. However, once we add a CPU-intensive postprocessor, one which may take longer to process a block than was required to collect it, then such a program must run asynchronously with the role-scanning loop, and there must be a provision for that loop to suspend its operations to allow CPU time for the blocks to be postprocessed. This creates a multiprogramming situation, whether run under an existing operating system or under a resource-sharing system built into the applications program.

Gregg (1977) emphasized that even seemingly simple protocols often have asynchronous, multitasking demands lurking beneath the surface. Any program that must process two independent external events must provide for the possibilities that one will be first, the other will be first, or they will be simultaneous to within the resolution of the computer. Gregg's remarks are as applicable to microprocessors as to minicomputers. In hardware terms, microprocessors are inexpensive, reliable, small, reasonably fast devices. They seem like the ideal bases for building intelligent data-logging systems.

On the surface, data logging is a simple linear task. Data flows in one end of the system from transducers and out the other end onto recorders, with a simple control program sitting in the middle. Unfortunately, any reasonable definition of intelligence makes the control program nonlinear. Intelligence is needed to cope with situations such as inconsistent data arrival and recording rates, and real-time inspection of the data being collected, with action being taken on the basis of that inspection. In such a system, several events are happening simultaneously. Direct memory access, multiprogramming on a hardware level, is only half of the solution; the system described above adds a software multiprogramming level to complete the solution.

The operating system not only shares resources among users, it allocates them within one user's protocol, so that high- and low-priority tasks can be separated, programs can run in synchrony or asynchrony as desired, and different experiments can share a common base of utility code. A failure to think in multiprogramming terms may seriously limit the amount of intelligence that can be built into our laboratories.

\section{REFERENCES}

GREGG, L. W. Maximizing the mini-uses of on-line computers. Behavior Research Methods \& Instrumentation, 1977, 9, 67.71.

TAYLOR, R. W. Spooling. In A. Ralston \& C. L. Meek (Eds.), Encyclopedia of computer science. New York: Petrocelli/ Charter, 1976. 